\title{
Effect of Mineralocorticoid Receptor Antagonism and ACE Inhibition on Angiotensin Profiles in Diabetic Kidney Disease: An Exploratory Study
}

\author{
Johannes J. Kovarik (D) - Christopher C. Kaltenecker • Oliver Domenig • \\ Marlies Antlanger · Marko Poglitsch · Chantal Kopecky • \\ Marcus D. Säemann
}

Received: May 29, 2021 / Accepted: July 7, 2021 / Published online: August 5, 2021

(c) The Author(s) 2021

\section{ABSTRACT}

Background: Renin-angiotensin-aldosterone system (RAAS) blockade with angiotensin-converting enzyme inhibitors (ACEi) or angiotensin

Chantal Kopecky and Marcus D. Säemann are equally contributing authors.

Supplementary Information The online version contains supplementary material available at https:// doi.org/10.1007/s13300-021-01118-7.

J. J. Kovarik (ه)

Division of Nephrology and Dialysis, Department of Internal Medicine III, Medical University Vienna, Währinger Gürtel 18-20, 1090 Vienna, Austria e-mail: johannes.kovarik@meduniwien.ac.at

\section{C. Kaltenecker}

Department of Pathology, Medical University

Vienna, Vienna, Austria

O. Domenig · M. Poglitsch

Attoquant Diagnostics GmbH, Vienna, Austria

\section{Antlanger}

2nd Department of Internal Medicine, Kepler

University Hospital, Med Campus III, Linz, Austria

C. Kopecky

School of Medical Sciences, Faculty of Medicine,

University of New South Wales, Sydney, Australia

M. D. Säemann

6th Medical Department with Nephrology and

Dialysis, Clinic Ottakring, Vienna, Austria

M. D. Säemann

Sigmund-Freud University, Vienna, Austria receptor blockers (ARB) is the cornerstone of antihypertensive treatment in patients with chronic kidney disease (CKD) and diabetes mellitus. Mineralocorticoid receptor antagonists (MRA) on top of conventional RAAS blockade confer cardio- and renoprotective effects. Yet, the detailed effects of this therapeutic approach on key RAAS effectors have not been elucidated to date.

Methods: In this exploratory placebo-controlled study, 15 patients with CKD stages 2-3 and albuminuria due to diabetic kidney disease (DKD) were randomized to receive the MRA eplerenone or placebo in addition to ACEi therapy. Employing mass-spectrometry, we quantified plasma angiotensin levels [Ang I, Ang II, Ang-(1-7), Ang-(1-5), Ang III, Ang IV], renin and aldosterone in patients before and after 8 weeks of MRA treatment.

Results: While blood pressure and kidney function were similar in the placebo and eplerenone treatment group during the study period, distinct differences in RAAS regulation occurred: eplerenone treatment resulted in an increase in plasma renin activity, Ang I and aldosterone concentrations, indicating global RAAS activation. In addition, eplerenone on top of ACEi profoundly upregulated the alternative RAAS effector Ang-(1-7).

Conclusions: Combined eplerenone and ACEi therapy increases Ang-(1-7) levels in patients with CKD indicating a unique nephroprotective RAAS pattern with considerable therapeutic implications. 
Keywords: Angiotensin; Aldosterone; Diabetes mellitus; Mineralocorticoid receptor antagonist; Renin; Renin-angiotensin-aldosterone system

\section{Key Summary Points}

There is an alarming rise of the prevalence of patients with chronic kidney disease (CKD) and diabetes mellitus globally, which is associated with excessive morbidity and mortality.

RAAS blockade with angiotensinconverting enzyme inhibitors (ACEi) or angiotensin receptor blockers (ARB) can dampen but not halt the progression of CKD.

We hypothesize that mineralocorticoid receptor antagonists (MRA) on top of conventional RAAS blockade change the angiotensin pattern resulting in cardioand renoprotective effects.

Eplerenone on top of ACEi upregulates plasma renin, Ang I and aldosterone concentrations, while Ang II remains suppressed.

Eplerenone add on to ACEi further increases plasma Ang-(1-7) levels.

Potential beneficial effects of alternative RAAS activation should be considered for personalised renoprotective therapies in patients with CKD and diabetes mellitus.

\section{INTRODUCTION}

The renin-angiotensin-aldosterone system (RAAS) is a complex regulatory network of peptides with widespread effects on blood pressure regulation, salt balance and fluid homeostasis [1-3]. RAAS blockade with ACE inhibitors (ACEi) or angiotensin receptor blockers (ARB) has been the cornerstone of antihypertensive therapy aimed at delaying progression of chronic kidney disease (CKD) and reducing proteinuria in both diabetic and non-diabetic patients with CKD [4-7]. It has been suggested that in patients with CKD and advanced diabetic kidney disease (DKD), ACEi might be superior to ARB in lowering risk of allcause mortality $[8,9]$. During the past decade, numerous animal and human studies have shown the beneficial effects of the selective mineralocorticoid receptor antagonist (MRA) eplerenone on renal outcome parameters like proteinuria $[10,11]$. Moreover, eplerenone treatment had anti-inflammatory, antifibrotic and antioxidative effects improving glomerular, arteriolar and tubulointerstitial lesions in rodent models, despite having no effect on haemodynamics, thereby securing nephroprotection $[12,13]$. Patients with CKD and diabetes mellitus have an enhanced susceptibility to adverse cardiovascular outcomes; hence these patients theoretically stand to benefit the most from add-on MRA therapy. The combination of eplerenone with ACEi has been shown to be beneficial in patients with chronic heart failure [14]. Furthermore, this combination therapy has been demonstrated to slow DKD progression in a rodent model [15] and to have additional anti-proteinuric effects in patients with CKD and type 2 diabetes mellitus (T2DM) $[10,14,16,17]$. Besides functional consequences, addition of MRA to ACEi might fundamentally affect the RAAS at the molecular level. Although often combined with RAAS blockers, the detailed effects of this therapeutic approach on RAAS metabolism have not been elucidated.

Accumulating evidence indicates that Ang(1-7), the key alternative RAAS effector peptide may confer beneficial vasodilatory, anti-inflammatory and antifibrotic effects opposing the actions of the 'classical RAAS' with its main effector Ang II [18-23]. Therefore, in this study we have investigated the effects of eplerenone on top of ACEi therapy compared to placebo on systemic RAAS metabolism with its key effector molecules.

Using a mass spectrometry-based approach [24], we have analysed quantitative differences between key Ang profiles of patients with CKD and diabetes mellitus receiving eplerenone on 
top of ACEi compared to placebo-treated patients in a prospective randomized trial.

\section{METHODS}

\section{Patient Recruitment}

This 10-week prospective, randomized, controlled exploratory trial included 15 patients with diabetes mellitus and proteinuric CKD. Patients had a mean HbA1c of $6.9 \pm 1.6 \%$ and a diagnosis of diabetes mellitus for a median 14.5 (IQR [7-23]) years. Nine of 15 patients had insulin dependence.

Patients were eligible for inclusion if they were diagnosed with albuminuric CKD stage 2-3 (defined by an estimated glomerular filtration rate (eGFR) (CKD-EPI) of $30-90 \mathrm{ml} / \mathrm{min} /$ $1.73 \mathrm{~m}^{2}$ ) and diagnosed T2DM (defined by World Health Organization (WHO) criteria). Proteinuria was defined by urinary albumin-tocreatinine ratio (UACR) $>300 \mathrm{mg} / \mathrm{g}$ on a spot urine sample or $>200 \mathrm{mg} / \mathrm{g}$ if they already received RAAS blockade with an ACEi or ARB [25]. Additionally, patients had to be neither hypo- nor severely hypertensive at the time of inclusion (systolic blood pressure $>120 \mathrm{mmHg}$ and $<180 \mathrm{mmHg}$ on ambulatory measurement). According to the Seventh Report of the Joint National Committee on Prevention, Detection, Evaluation, and Treatment of High Blood Pressure (JNC7) classification, all patients classified as having high normal blood pressure or stage 1 or 2 hypertension [26].

Exclusion criteria were as follows: age $<18$ years, $>80$ years, UACR $>3500 \mathrm{mg} / \mathrm{g}$, severe hypertension, pregnancy (or planned pregnancy), coronary heart disease, severe renal insufficiency $\left(\right.$ eGFR $<30 \mathrm{ml} / \mathrm{min} / 1,73 \mathrm{~m}^{2}$ ) systolic blood pressure $<120 \mathrm{mmHg}$, additional RAAS interfering drugs (ACEi, ARB, direct renin inhibitors), 25-hydroxy vitamin $\mathrm{D}$ levels below $16.6 \pm 8.3 \mathrm{pg} / \mathrm{ml}, \quad 1,25$-dihydroxy vitamin $\mathrm{D}$ $33.1 \pm 15.5 \mathrm{pg} / \mathrm{ml}$, severe liver insufficiency (Child-Pugh class C), potassium sparing diuretics, strong CYP3A4-inhibitors, serum potassium level $>5 \mathrm{mmol} / \mathrm{L}$ at study inclusion, intolerance to eplerenone or an excipient of it.
All patients were recruited from the outpatient clinic of the Clinical Division of Nephrology and Dialysis of the Medical University of Vienna, Austria between 2013 and 2017. We had a total dropout of four patients (two because of non-compliant study medication intake, two because of suspected unexpected serious adverse reaction (hospitalisation during study period).

\section{Study Procedures}

This study was approved by the Ethics Committee of the Medical University of Vienna (EK 1329/2012). It was registered at the European Clinical Trials Database (EUDRACT No. 2012-002175-34) and at a publicly available clinical trial database [https://clinicaltrials.gov (NCT01832558)]. The study was carried out according to good clinical and scientific practice guidelines and in accordance with the Helsinki Declaration of 1964, and its later amendments. All participants provided their written informed consent prior to participation.

All study participants underwent a 2-week run-in phase to unify their ACEi therapy to enalapril (20 mg daily). Blood collection was then performed for baseline RAAS analysis, measurement of renin and aldosterone as well as a urine analysis for albuminuria. If severely hypertensive blood pressure values were elicited through this measure, additional antihypertensive treatment, preferably with calcium channel blockers or beta-blockers were introduced.

Subsequently, patients were randomized to either receive eplerenone $25 \mathrm{mg}$ (Fig. 1 and Supplementary Fig. 1) or placebo on top of ACEi for 8 weeks. Patients were instructed to take the study medication $2 \mathrm{~h}$ before blood collection. A routine laboratory control of electrolytes and creatinine was performed after the first week of the study phase. At the end of the active study phase, analysis of RAAS components as well as routine blood analysis was performed again. After the end of the study phase (after 8 weeks), patients continued to be treated at the outpatient clinic of the Clinical Division of Nephrology according to good clinical practice guidelines. Continuation or discontinuation of 


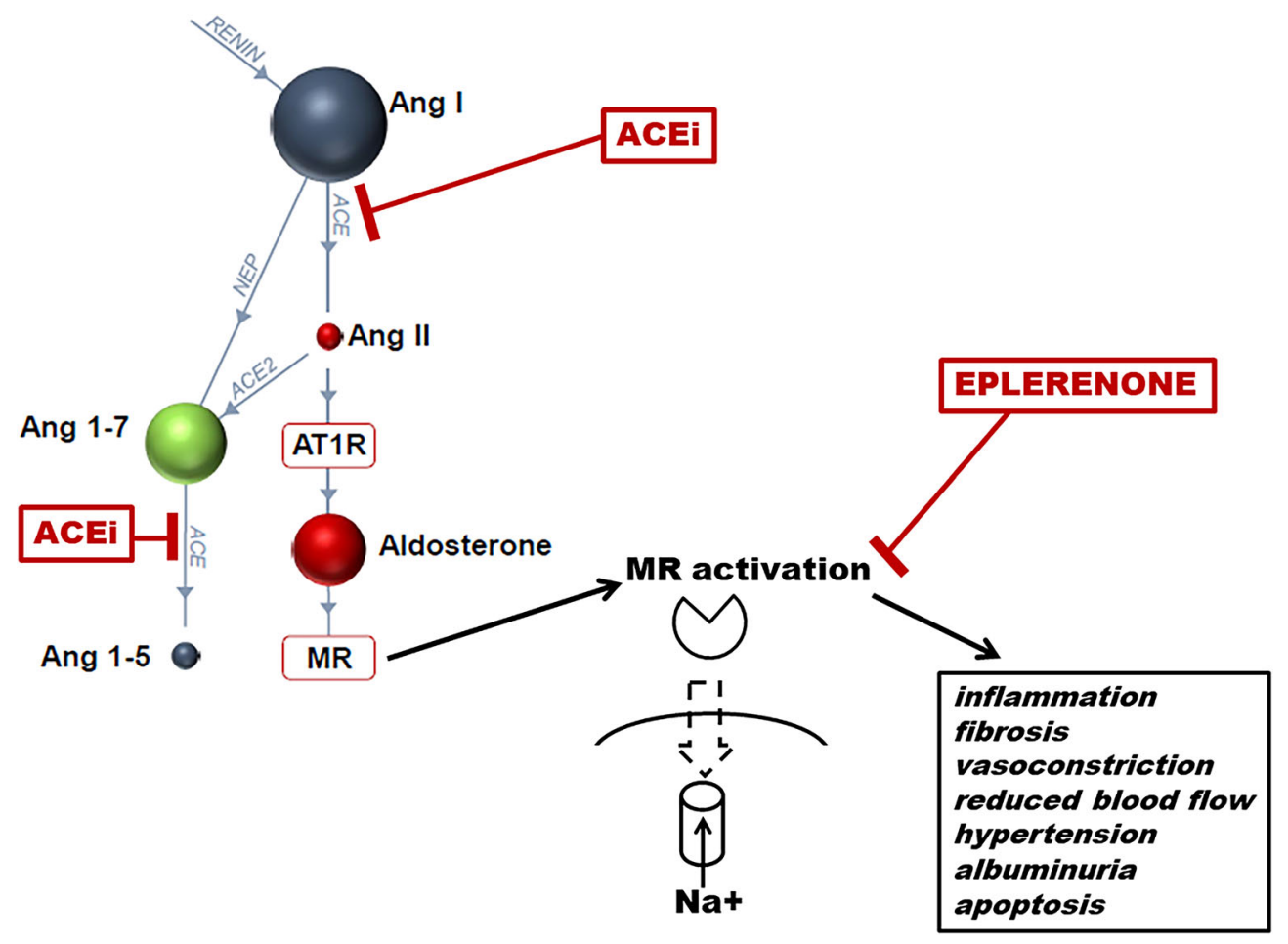

Fig. 1 Schematic model of the RAAS affected by MRA (eplerenone) and ACEi. RAAS enzymes, angiotensins and interfering substances used in the study are displayed in the figure. Eplerenone on top of ACEi upregulates renin,

eplerenone treatment after the end of the study was the choice of the medical team of the outpatient clinic.

\section{Blood Collection and Processing}

For the quantification of angiotensin metabolites and aldosterone, a blood collection via cubital vein was performed. Six millilitres of heparinized peripheral blood was collected. Plasma was obtained by centrifugation $\left(4^{\circ} \mathrm{C}\right)$ at $2000 \times g$ for $10 \mathrm{~min}$ and 2 -ml aliquots were stored at $-80{ }^{\circ} \mathrm{C}$ until analysis. Equilibrium concentrations of the peptides Ang I, Ang II, Ang III, Ang IV, Ang-(1-7) and Ang-(1-5), as well as the level of the steroid aldosterone, were measured in human plasma by LC-MS/MS (Attoquant Diagnostics, Vienna). Briefly, samples were spiked with stable isotope-labelled (peptides) or deuterated (aldosterone) internal standards after equilibration [27] and analytes
Ang I and Ang-(1-7) and aldosterone while Ang II remains suppressed. This therapeutic interference might act synergistically to inhibit the detrimental effects of MR activation

were extracted using C18-based solid-phase extraction. Extracted samples were analysed using mass spectrometry analysis using a reversed-phase analytical column (Acquity UPLC C18, Waters) operating in line with a XEVO TQ-S triple quadrupole mass spectrometer (Waters Xevo TQ/S, Milford, MA) in multiple reaction monitoring mode. Internal standards were used to correct for analyte recovery across the sample preparation procedure in each individual sample. Analyte concentrations were calculated from integrated chromatograms considering the corresponding response factors determined in appropriate calibration curves in plasma matrix, when integrated signals exceeded a signal-to-noise ratio of 10 . The lower limits of quantification were $3 \mathrm{pmol} / \mathrm{l}$; (Ang I), 2 pmol/l (Ang II), 2.5 pmol/l (Ang III), 2 pmol/1 (Ang IV), 3 pmol/1 (Ang-(1-7)), 2 pmol/l (Ang(1-5)) and $20 \mathrm{pmol} / \mathrm{l}$ (aldosterone), respectively. 
Table 1 Demographic and baseline values at study medication start (after 2 weeks run-in phase)

\begin{tabular}{llll}
\hline Variable & Placebo $(\boldsymbol{n}=\mathbf{8})$ & Eplerenone $(\boldsymbol{n}=7)$ & $\boldsymbol{p}$ value \\
\hline Female sex, $n$ (\%) & $3(37.5)$ & $0(0)$ & 0.079 \\
Age, years & $56 \pm 10$ & $63 \pm 9$ & 0.162 \\
Body mass index, $\mathrm{kg} / \mathrm{m}^{2}$ & $30.5 \pm 8.8$ & $27.8 \pm 1.8$ & 0.523 \\
Serum creatinine $\mathrm{mg} / \mathrm{dl}$ & $1.49 \pm 0.53$ & $1.97 \pm 0.43$ & 0.080 \\
eGFR, $\mathrm{ml} / \mathrm{min} / 1.73 \mathrm{~m}^{2}$ & $61.7[35.7-96.3]$ & $42.3[32.5-57.5]$ & 0.328 \\
Glucose, mg/dl & $136 \pm 75$ & $142 \pm 35$ & 0.843 \\
HbAlc \% & $7.2 \pm 0.6$ & $6.5 \pm 0.5$ & 0.416 \\
UPCR, mg/g & $1253 \pm 755$ & $1388 \pm 923$ & 0.761 \\
UACR, mg/g & $1000 \pm 592$ & $1142 \pm 735$ & 0.709 \\
RR systolic, mmHg & $139 \pm 17$ & $151 \pm 18$ & 0.247 \\
RR diastolic, mmHg & $83 \pm 9$ & $82 \pm 5$ & 0.823 \\
PRA-S, pmol/l & $222.5[92.6-1701]$ & $73.50[33.4-799.0]$ & 0.593 \\
ACE-S, $(\mathrm{pmol} / \mathrm{l}) /(\mathrm{pmol} / \mathrm{l})$ & $0.01[0.01-0.03]$ & $0.04[0.02-0.04]$ & 0.454 \\
\hline
\end{tabular}

Data are shown as mean \pm SD or median [interquartile range]

$e G F R$ estimated glomerular filtration rate, UPCR urinary protein-to-creatinine ratio, $U A C R$ urine albumin-to-creatinine ratio, $P R A$ plasma renin activity, $A C E$ angiotensin-converting enzyme

Angiotensin-based markers for renin (PRA-S) and angiotensin-converting enzyme (ACE-S) were derived from Ang II and Ang I levels by calculating their sum and their ratio, respectively $[28,29]$. Additionally, direct renin concentration was measured with a clinical chemiluminescence immunoassay (Diasorin, Saluggia, Italy).

\section{Statistical Analysis}

Demographic data are presented as mean values with standard deviation (SD) if normally distributed (verified by Shapiro-Wilk's test) and unpaired Student's $t$ test was performed to determine differences between treatment and placebo groups. Non-parametric data are shown as medians [interquartile range] and Mann-Whitney's $U$ test was applied to determine differences between treatment and placebo groups.
Angiotensin values below the lower limit of quantification (LLOQ) were substituted by LLOQ/Sqrt(2) [30, 31]. In Fig. 4, calculated median values, which were below the LLOQ, were specified as such. For the analysis of angiotensins before and after medical intervention, Wilcoxon's signed-rank test was used. A $p$ value of 0.05 or less was considered statistically significant. IBM SPSS System version 20.0.0 (SPSS, Inc., 2010, Chicago, IL) and GraphPad Prism Version 6 were used for all analyses (San Diego, CA).

\section{RESULTS}

\section{Baseline Clinical Characteristics}

Both study groups were comparable in demographic and baseline laboratory characteristics at the time of study inclusion (Table 1 and Supplementary Table 1). Of note, serum 
creatinine levels of the eplerenone group were significantly higher at inclusion; however, no differences in eGFR were observed. Three patients in the placebo group received additional antihypertensive therapy (beta-blocker and/or calcium antagonist) and one patient in the treatment group received a beta-blocker in addition to ACEi therapy. Therapy adherence of all study patients was assessed after the 2 -week run-in phase and at the end of the active study phase by mass spectrometry-based measurements of enalaprilat levels (data not shown). All patients had previously received dietary counselling addressing the requirements of CKD stages 2-3.
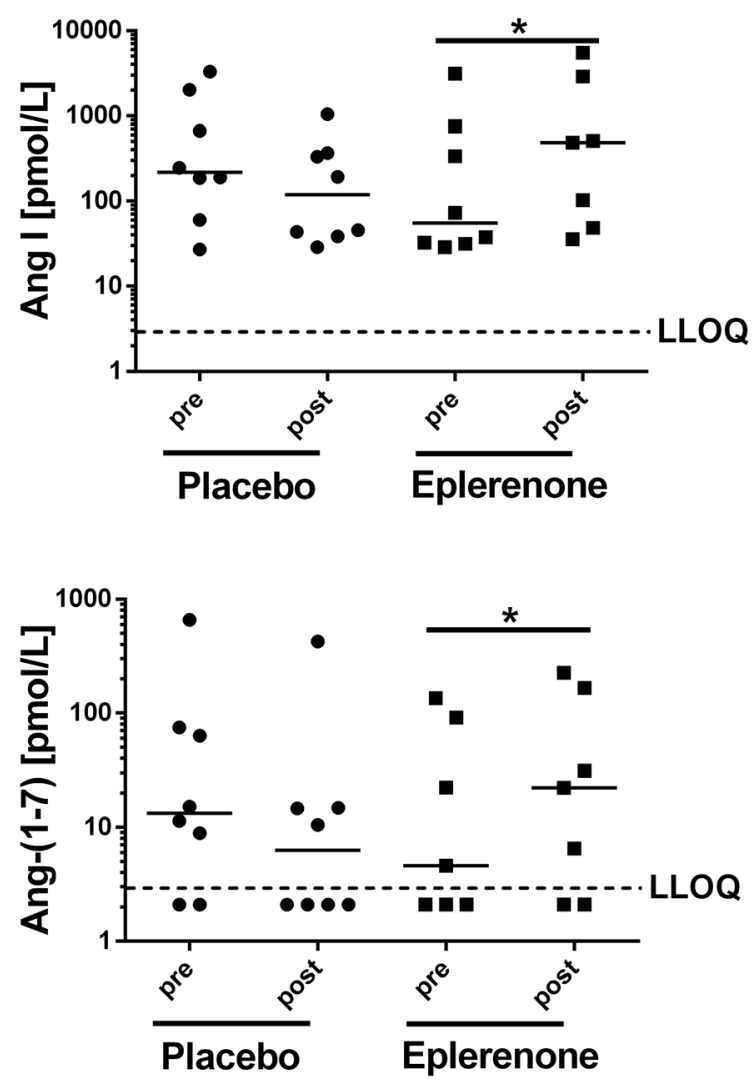

Fig. 2 Classical and alternative RAAS metabolites after eplerenone treatment. Ang I, Ang II, Ang-(1-7) and aldosterone levels are shown before and after 8 weeks of eplerenone or placebo treatment in patients with $\mathrm{CKD}$
Eplerenone on top of ACEi Upregulates Ang I, Ang-(1-7) and Aldosterone

While we found Ang II to be similarly suppressed in the eplerenone (median 3.0 [IQR $2.7-37.5 \mathrm{pmol} / \mathrm{l}])$ and placebo group (2.6 [1.4-$11.8 \mathrm{pmol} / \mathrm{l}])$, we observed a significant Ang I upregulation after eplerenone treatment (median 487.6 [48.3-2894.2 pmol/l]) (Fig. 2) compared to placebo (median 119.3 [39.7-359.1 pmol/1], $(p<0.05))$. The key effector of the alternative RAAS axis Ang-(1-7) was also significantly upregulated after eplerenone treatment (median 22.2 [2.1-166.1 pmol/1]) versus placebo (median 6.3 [2.1-14.8 pmol/l], $(p<0.05))$, while downstream RAAS effectors such as Ang-(1-5), Ang III and Ang IV remained close to or below the lower limit of quantitation
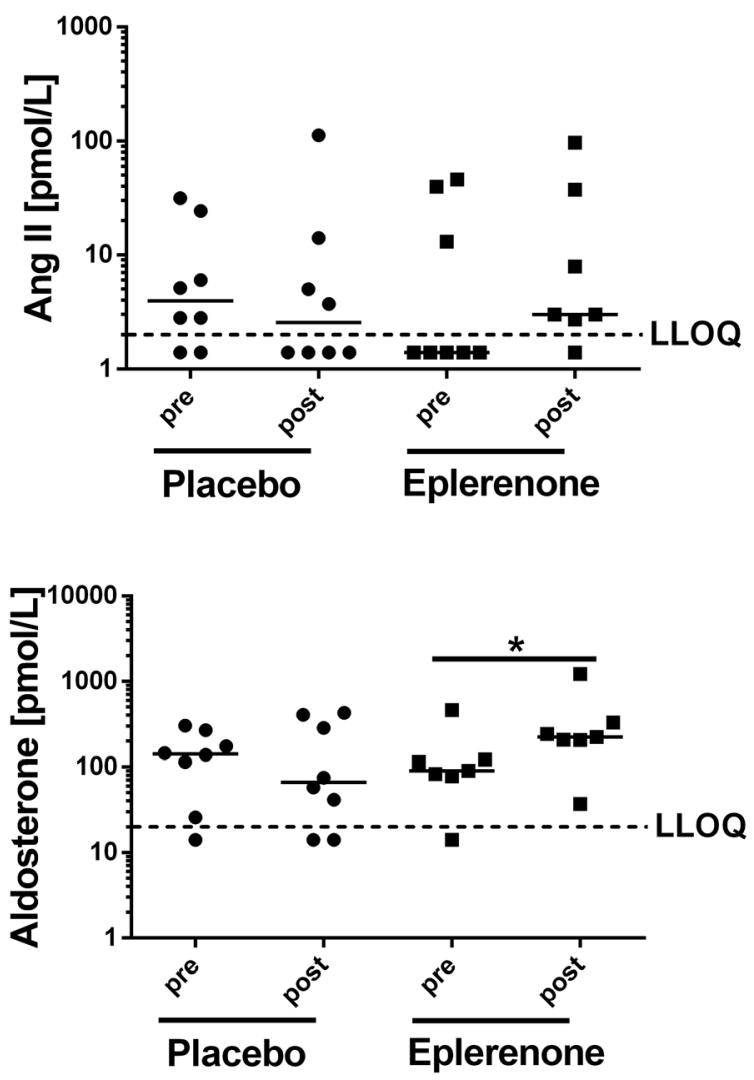

and T2DM. Concentrations are given in pmol/l. ${ }^{*} p<0.05$. For better visualization data are plotted logarithmically on the $y$-axis 


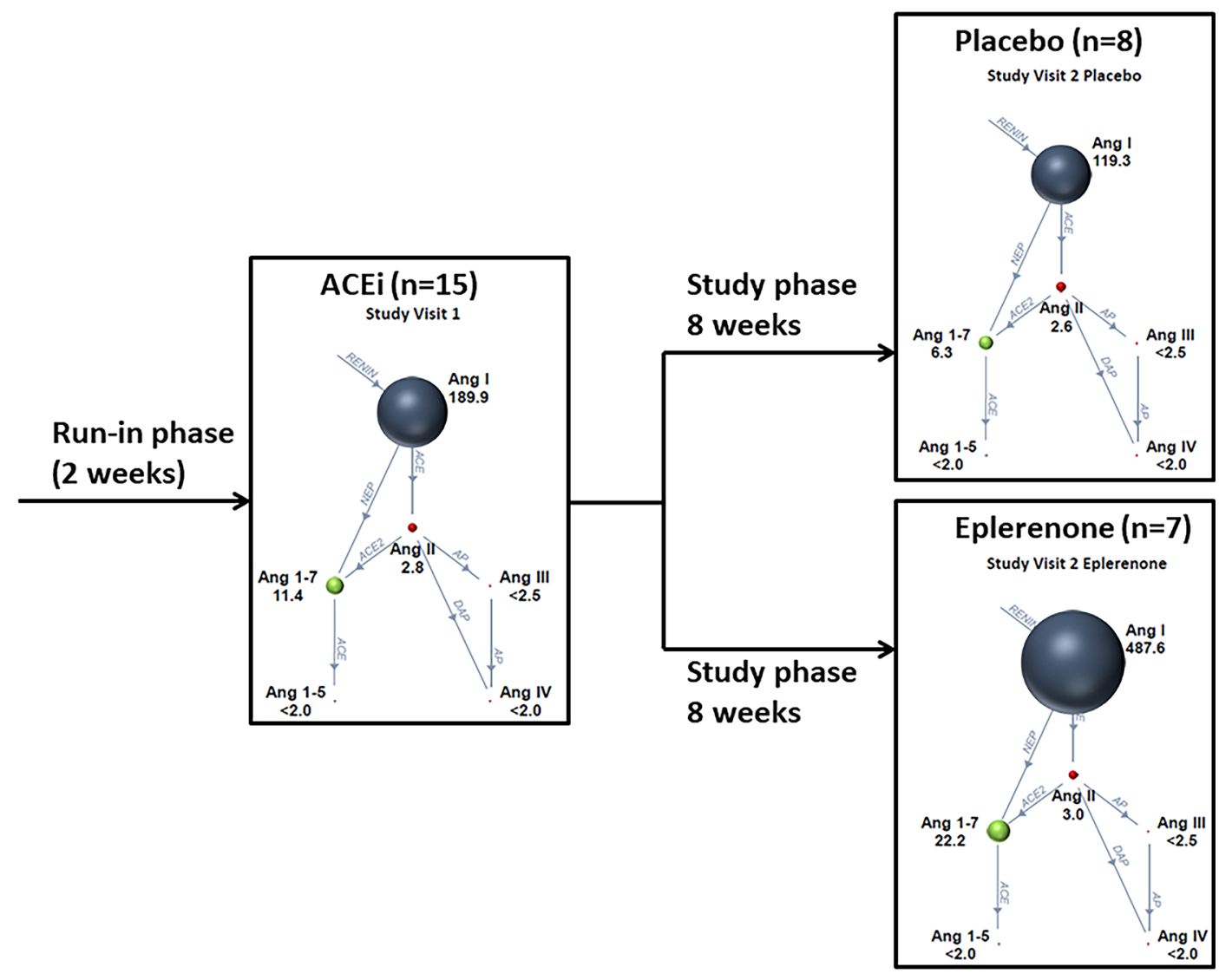

Fig. 3 Group median angiotensin levels of the study cohort. The illustration on the left shows the median Ang values of all 15 patients after the run-in phase, during which unification to the ACEi enalapril occurred. The right upper illustration shows median Ang values after additional placebo intake; the right lower illustration

(LLOQ $<2.0 \mathrm{pmol} / \mathrm{l})$ in both study groups (Fig. 3 and Table 2). Individual angiotensin levels before and after placebo or eplerenone treatment are shown in Supplementary Fig. 3.

\section{Eplerenone on top of ACEi Upregulates Renin While ACE Activity Remains Suppressed}

Both renin and aldosterone concentrations were prominently affected by eplerenone treatment, with eplerenone treatment inducing a seven-fold increase in PRA-S (angiotensin-based marker for renin (calculated by sum of Ang I + Ang II) [28] (median 73.5 [IQR shows median values after additional eplerenone intake. Size of spheres and numbers represent median Ang concentrations in pmol/l analysed by mass spectrometry directly from each patient's plasma. Median values, which were below the lower limit of quantification (LLOQ), were specified as $<2.0 ;<2.5$

33.4-799.0 pmol/l] before treatment vs. 495.5 [49.3-2932 pmol/l] after treatment; $p<0.05$ ), while PRA-S remained stable in the placebo group during the treatment period (222.5 [92.6-1701 $\mathrm{pmol} / \mathrm{l}]$ before treatment vs. 176.2 [40.7-363.1 pmol/1] after treatment; $p=0.161$ ) (Fig. 4). Direct renin concentrations showed only a moderate correlation with plasma renin activity (Supplementary Fig. 2). However, the observed increase in plasma renin in the eplerenone group remained significant. Similarly, plasma aldosterone concentration was upregulated following eplerenone treatment (median 90.2 [IQR 78.0-122.7 pmol/l] before treatment vs. 222.2 [206.1-329.9 pmol/l] after treatment; 
Table 2 Angiotensin concentrations before and after medication

\begin{tabular}{|c|c|c|c|c|c|c|}
\hline \multirow{2}{*}{$\begin{array}{l}\text { Variable } \\
\text { (pmol/l) }\end{array}$} & \multicolumn{3}{|l|}{ Placebo } & \multicolumn{3}{|l|}{ Eplerenone } \\
\hline & Baseline & 8 weeks eplerenone & $p$ value & Baseline & 8 weeks eplerenone & $p$ value \\
\hline Ang I & $218.1[91.6-1676]$ & $119.3[39.7-359.1]$ & 0.123 & $72.5[32.4-752.9]$ & $487.6[48.3-2894.2]$ & $0.018^{*}$ \\
\hline Ang II & $4.0[1.8-19.7]$ & $2.6[1.4-11.8]$ & 0.498 & $1.4[1.4-39.8]$ & $3.0[2.7-37.5]$ & 0.752 \\
\hline Ang-(1-7) & $13.3[3.8-71.8]$ & $6.3[2.1-14.8]$ & 0.075 & $4.6[2.1-91.5]$ & $22.2[2.1-166.1]$ & $0.043^{*}$ \\
\hline Ang-(1-5) & $1.4[1.4-1.4]$ & $1.4[1.4-1.4]$ & 0.317 & $1.4[1.4-1.4]$ & $1.4[1.4-1.4]$ & 0.655 \\
\hline Ang III & $1.8[1.8-1.8]$ & $1.8[1.8-1.8]$ & 1.0 & $1.8[1.8-1.8]$ & $1.8[1.8-1.8]$ & 1.0 \\
\hline Ang IV & $1.4[1.4-1.4]$ & $1.4[1.4-1.4]$ & 0.317 & $1.4[1.4-1.4]$ & $1.4[1.4-1.4]$ & 0.317 \\
\hline PRA-S & $222.5[92.6-1701]$ & $176.2[40.7-363.1]$ & 0.161 & $73.50[33.4-799.0]$ & $495.5[49.3-2932]$ & $0.018^{*}$ \\
\hline Aldosterone & $142.0[47.9-245.0]$ & $66.5[21.0-376.7]$ & 0.866 & $90.2[78.0-122.7]$ & 222.2 [206.1-329.9] & $0.018^{*}$ \\
\hline
\end{tabular}

Ang angiotensin

${ }^{*} p<0.05$

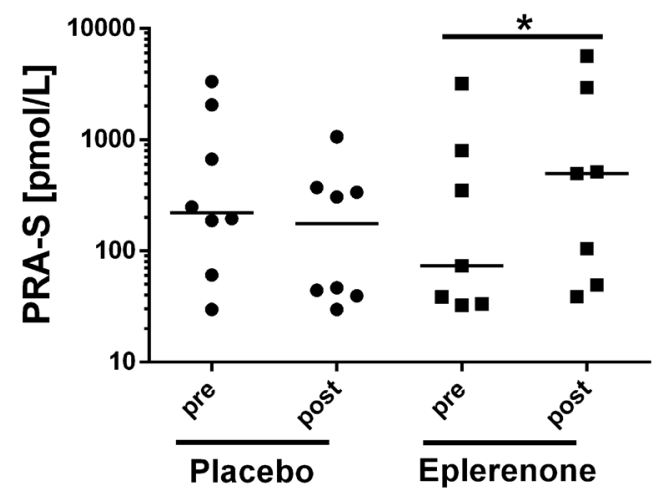

Fig. 4 Renin and ACE activity. PRA-S (marker for renin activity) and ACE-S (marker for ACE activity) before and after 8 weeks of eplerenone or placebo treatment in

$p<0.05)$, while aldosterone concentrations remained stable in the placebo group (median 142.0 [47.9-245.0 pmol/l] before treatment vs. 66.5 [21.0-376.7 pmol/l] after treatment; $p=0.87)$. Individual aldosterone, PRA-S and ACE-S levels before and after placebo or eplerenone treatment are shown in Supplementary Fig. 4. In both patient groups, plasma ACE activity (ACE-S, calculated by ratio Ang II/Ang I [29]) was not significantly affected by eplerenone treatment (median 0.02 [0.01-0.03] compared to placebo (0.03 [IQR 0.01-0.05]; $p=0.411)$. To further analyse the main

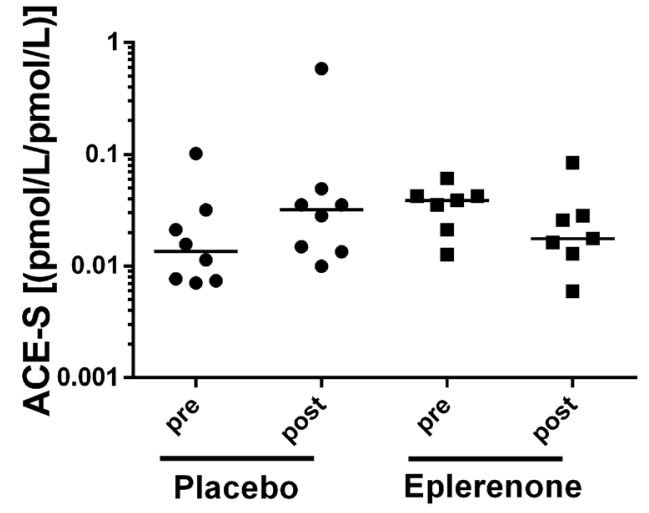

patients with CKD and T2DM. ${ }^{*} p<0.05$. For better visualization data are plotted logarithmically on the $y$-axis

enzymes responsible for Ang-(1-7) synthesis we measured plasma ACE2 levels and calculated the Ang-(1-7)/Ang I ratio representing neprilysin (NEP) activity. We found that neither ACE2 levels nor Ang-(1-7)/Ang I ratios were different between the placebo and eplerenone group (Fig. 5).

\section{Blood Pressure, Kidney Function and Proteinuria}

There were no significant differences in arterial blood pressure decrease between the eplerenone 


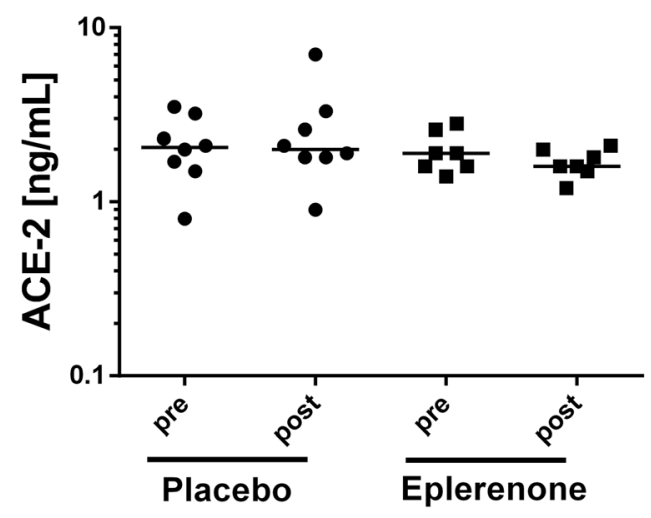

Fig. 5 ACE2 and Ang-(1-7)/Ang I ratio. ACE2 and Ang(1-7)/Ang I ratio (marker for NEP activity) before and after 8 weeks of eplerenone or placebo treatment in

and the placebo group (Table 3 ). We observed no change in eGFR (median 42 [32-57] $\mathrm{ml} / \mathrm{min} /$ $1.73 \mathrm{~m}^{2}$ before treatment vs. 41 [29-52-] $\mathrm{ml} /$ $\mathrm{min} / 1.73 \mathrm{~m}^{2}$ after treatment) and proteinuria (urinary protein/creatinine ratio (UPCR)) (median $1388 \pm 923 \mathrm{mg} / \mathrm{g}$ before treatment vs. $1041 \pm 63 \mathrm{mg} / \mathrm{g}$ after treatment) within the eplerenone group. Similarly, no change in the placebo group was observed. As measures of patient safety, serum potassium and eGFR were measured regularly. At 1 week of eplerenone treatment, potassium levels increased from $4.5 \pm 0.3 \mathrm{mmol} / \mathrm{l} \quad$ to $\quad 4.8 \pm 0.3 \mathrm{mmol} / \mathrm{l}$ $(p<0.05)$, while eGFR remained stable $(42$ [33-56] $\mathrm{ml} / \mathrm{min} / 1.73 \mathrm{~m}^{2}$ vs. 37 [30-43] $\mathrm{ml} / \mathrm{min} /$ $1.73 \mathrm{~m}^{2}$ ). At the end of the 8-week study phase, plasma potassium increased to $5.1 \pm 0.5 \mathrm{mmol} /$ 1 in the eplerenone group and remained stable in the placebo group $4.5 \pm 0.4 \mathrm{mmol} / \mathrm{l}$.

\section{DISCUSSION}

The underlying molecular mechanisms of the renoprotective effects of MRA in patients with DKD remain largely unknown. In this placebocontrolled study we have found a distinct molecular response of the RAAS reflected by upregulation of renin, Ang I and high systemic Ang-(1-7) levels while Ang II remained suppressed in DKD patients receiving 8 weeks of

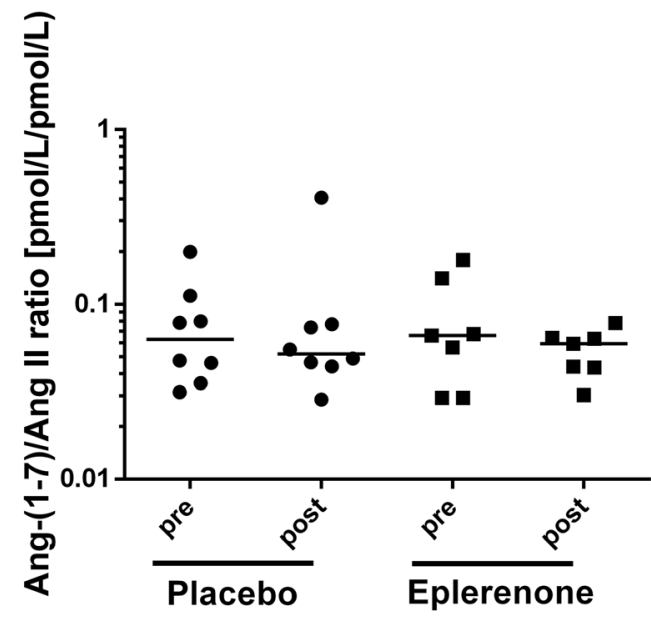

patients with CKD and T2DM. For better visualization data are plotted logarithmically on the $y$-axis

treatment with the MRA eplerenone in addition to ACEi, indicative of a superior nephroprotective angiotensin pattern.

The overall effect on blood pressure and kidney function was comparable in both study groups. At the molecular level, eplerenone treatment activated the systemic RAAS reflected by upregulation of renin and Ang I, while Ang II remained suppressed. Interestingly, the key effector molecule of the alternative RAAS, Ang(1-7), was significantly upregulated by eplerenone possibly contributing to the observed beneficial clinical outcomes following this treatment in these patients [11].

RAAS blockade with ACEi has been the cornerstone for antihypertensive treatment of patients with CKD and diabetes mellitus [4, 5]. While it has been shown in some studies that ACEi were superior to ARB regarding cardiovascular death and all-cause mortality in patients with CKD and DKD [8], there still remains a substantial residual renal risk, favouring add-on therapy such as MRA.

We have previously shown that beneficial effects associated with ACEi use are not solely mediated by the reduction in blood pressure and decrease in glomerular pressure, but also by fundamental changes of RAAS effector peptide profiles [32, 33]. Following RAAS blockade with ACEi an upregulation of renin and Ang I via a negative feedback mechanism has been 
Table 3 Secondary outcome parameters

\begin{tabular}{llll}
\hline Variable & Placebo $(\boldsymbol{n}=\mathbf{8})$ & Eplerenone $(\boldsymbol{n}=7)$ & $\boldsymbol{p}$ value \\
\hline$\Delta$ Renin, $\mu \mathrm{IU} / \mathrm{ml}$ & $-25.4[-216.9$ to 15.5$]$ & $14.5[1.5-568.0]$ & 0.04 \\
$\Delta$ Aldosterone, pmol/l & $-14.5[-83.5$ to 104.3$]$ & $120.5129 .9[120.5-215.9]$ & 0.05 \\
$\Delta$ eGFR, $\mathrm{ml} / \mathrm{min} / 1.73 \mathrm{~m}^{2}$ & $-0.7 \pm 9.3$ & $-2.1 \pm 4.4$ & 0.738 \\
$\Delta$ UPCR, $\mathrm{mg} / \mathrm{g}$ & $86.50 \pm 298.4$ & $-346.1 \pm 443.6$ & 0.04 \\
$\Delta$ UACR, mg/g & $47.0[11.0-80.3]$ & $-75.0[-486.0$ to 324.0$]$ & 0.52 \\
$\Delta$ RR systolic, $\mathrm{mmHg}$ & $-3.0[-12.8$ to 22.8$]$ & $0.0[-9.0$ to 3.8] & 0.90 \\
$\Delta$ RR diastolic, mmHg & $-2.0[-6.8$ to 5.0$]$ & $5.5[-6.3$ to 15.8$]$ & 0.38 \\
$\Delta$ Potassium, mmol/l & $-0.06[-0.45$ to 0.16$]$ & $0.58[0.31-0.90]$ & 0.00 \\
$\Delta$ PRA-S, pmol/l & $565.1 \pm 921.9$ & $746.7 \pm 1058$ & 0.02 \\
$\Delta$ ACE-S, $(\mathrm{pmol} / \mathrm{l}) /(\mathrm{pmol} / \mathrm{l})$ & $0.07 \pm 0.20$ & $-0.01 \pm 0.03$ & 0.23 \\
\hline
\end{tabular}

$\triangle$ change from baseline, $e G F R$ estimated glomerular filtration rate, UPCR urinary protein-to-creatinine ratio, $U A C R$ urinary albumin-to-creatinine ratio, $R R$ blood pressure

previously described [27, 32]. Importantly, therapeutic ACE inhibition suppresses pro-inflammatory and pro-fibrotic plasma Ang II levels and upregulates plasma Ang-(1-7), which might confer various renoprotective effects $[32,33]$.

MRA treatment has beneficial effects in animal models of DKD and has been shown to lower the risk of CKD progression and cardiovascular events $[11,13]$. Specifically eplerenone administration reduced proteinuria and glomerular, arteriolar and tubulointerstitial lesions in the kidney, despite having no effect on haemodynamics and fewer clinical side effects compared to spironolactone [13].

Here, we combine ACEi with MRA treatment and could show that eplerenone on top of ACEi further upregulates the nephroprotective RAAS effector Ang-(1-7). Accumulating data indicate that this angiotensin is centred in the alternative neprilysin (NEP)/angiotensin-converting enzyme 2 (ACE2)/angiotensin (Ang)-(1-7)/Mas receptor (MasR) axis, which has beneficial antiinflammatory, vasodilatatory and anti-proteinuric effects [21, 23, 34]. Hence, our results provide a strong incentive to further investigate Ang-(1-7) as a therapeutic target in the treatment of diabetes-induced hypertension and renal damage in human studies. While Ang-
(1-7) has no direct agonistic activity on aldosterone secretion, this heptapeptide has been reported to act as a negative modulator of aldosterone secretion in vitro and in vivo $[35,36]$.

As expected, eplerenone treatment neither affected Ang-(1-5), the product of ACE-driven Ang-(1-7) conversion, nor downstream products of Ang II such as Ang III and Ang IV.

It is known that MRA blockade upregulates plasma renin and aldosterone levels in patients with hypertension $[37,38]$. Our study therefore implies that treatment with eplerenone on top of ACEi results in additional proximal substrates for the RAAS cascade represented by increased renin activity (PRA-S) and Ang I levels. Furthermore, eplerenone on top of ACEi results in an upregulation of Ang-(1-7) while simultaneously suppressing Ang II.

However, ACE2 levels and the Ang-(1-7)/ Ang I ratio (reflecting NEP activity) remained similar in patients receiving eplerenone compared to placebo indicating no relative differences in enzyme activity for Ang-(1-7) formation. Therefore, it is tempting to speculate that the additional upregulation of Ang-(1-7) observed in our study might stem from overall RAAS activation, which is also reflected by 
elevated renin, PRA-S and Ang I concentrations in the eplerenone treatment group.

Our findings offer a reasonable targeted therapeutic strategy aimed at the alternative RAAS metabolism to enforce high plasma Ang(1-7) levels and to reduce the residual renal risk in patients with DKD under conventional RAAS inhibitor therapy.

Accumulating data obtained from prospective clinical trials have established that the risk for developing severe hyperkalemia in patients with CKD is significantly less prevalent than previously thought, and that instituting MRA in combination with RAAS blockade in these patients with appropriate laboratory surveillance may be a reasonable treatment option $[16,39]$. Furthermore, recent studies demonstrating the efficacy of novel non-absorbed, orally administered, potassium-binding polymers indicate that adequate surveillance, moderate or low-dose MRA, and use of these potassium-binding polymers may help to adequately control serum potassium in patients with CKD even in advanced CKD stages [40]. Therefore, successful RAAS blockade in patients with CKD can be employed when careful consideration to the individual patient characteristics along with consequent and careful monitoring of eGFR and serum-potassium is given.

Our study has some limitations: first, it is of exploratory design and the number of included patients is small. Second, of the 15 included patients, only 3 were women, precluding the potential to make a statement on the influence of gender on RAAS regulation. Third, there is no long-term (i.e. more than 3 months) follow-up data of the studied patients. Forth, no women were in the eplerenone subgroup.

Strengths of the study are its prospective, placebo-controlled nature, examination of human subjects and the simultaneous quantification of several RAAS metabolites by a highly selective and specific assay (internal standardization, UPLC-MS/MS analysis) $[24,41]$.

\section{CONCLUSIONS}

This is the first trial studying the molecular changes of both the classical and alternative RAAS under eplerenone treatment on top of ACEi in patients with CKD and T2DM. We demonstrate that eplerenone on top of ACEi further increases renin and Ang I levels and the key alternative RAAS peptide Ang-(1-7). As the alternative RAAS potentially confers nephroprotective effects, these findings might be taken into consideration in the treatment of patients with CKD and T2DM.

\section{ACKNOWLEDGEMENTS}

We want to thank the team members of the outpatient clinic of the Clinical Division of Nephrology and Dialysis of the Medical University Vienna for careful handling of blood and urine samples. The authors want to thank any study participant for their involvement in the study.

Funding. The journal's Rapid Service Fee was funded $50 \%$ by the authors and $50 \%$ by the Medical University of Vienna.

Authorship. All named authors meet the International Committee of Medical Journal Editors (ICMJE) criteria for authorship for this article, take responsibility for the integrity of the work as a whole, and have given their approval for this version to be published.

Authorship Contributions. Conceptualization: Marcus D. Säemann; Methodology: Marko Poglitsch, Oliver Domenig; Formal analysis and investigation: Johannes J. Kovarik; Writingoriginal draft preparation: Johannes J. Kovarik, Marlies Antlanger, Chantal Kopecky; Writingreview and editing: Christopher Kaltenecker; Supervision: Marcus D. Säemann. All authors read and approved the final manuscript.

Disclosures. Johannes J. Kovarik, Christopher C. Kaltenecker, Oliver Domenig, Marlies Antlanger, Marko Poglitsch, Chantal Kopecky 
and Marcus D. Säemann have nothing to disclose.

Compliance with Ethics Guidelines. This study was approved by the Ethics Committee of the Medical University of Vienna (EK 1329/2012). It was registered at the European Clinical Trials Database (EUDRACT No. 2012-002175-34) and at a publicly available clinical trial database (https://clinicaltrials.gov (NCT01832558)). The study was carried out according to good clinical and scientific practice guidelines and in accordance with the Helsinki Declaration of 1964, and its later amendments. All participants provided their written informed consent prior to participation.

Data Availability. The datasets generated and analysed during the current study are available from the corresponding author on reasonable request.

Open Access. This article is licensed under a Creative Commons Attribution-NonCommercial 4.0 International License, which permits any non-commercial use, sharing, adaptation, distribution and reproduction in any medium or format, as long as you give appropriate credit to the original author(s) and the source, provide a link to the Creative Commons licence, and indicate if changes were made. The images or other third party material in this article are included in the article's Creative Commons licence, unless indicated otherwise in a credit line to the material. If material is not included in the article's Creative Commons licence and your intended use is not permitted by statutory regulation or exceeds the permitted use, you will need to obtain permission directly from the copyright holder. To view a copy of this licence, visit http://creativecommons.org/licenses/by$\mathrm{nc} / 4.0 /$.

\section{REFERENCES}

1. Peach MJ. Renin-angiotensin system: biochemistry and mechanisms of action. Physiol Rev. 1977;57(2): 313-70.
2. Ito M, Oliverio MI, Mannon PJ, et al. Regulation of blood pressure by the type $1 \mathrm{~A}$ angiotensin II receptor gene. Proc Natl Acad Sci USA. 1995;92(8): 3521-5.

3. Lavoie JL, Sigmund CD. Minireview: overview of the renin-angiotensin system-an endocrine and paracrine system. Endocrinology. 2003;144(6): 2179-83.

4. Heeg JE, de Zeeuw D, de Jong PE. Antiproteinuric effect of ACE inhibitors. Lancet. 1988;2(8622): 1251-2.

5. Taguma Y, Kitamoto Y, Futaki G, et al. Effect of captopril on heavy proteinuria in azotemic diabetics. N Engl J Med. 1985;313(26):1617-20.

6. Remuzzi G, Ruggenenti P, Perico N. Chronic renal diseases: renoprotective benefits of renin-angiotensin system inhibition. Ann Intern Med. 2002;136(8):604-15.

7. Marquez DF, Ruiz-Hurtado G, Ruilope LM, Segura J. An update of the blockade of the renin angiotensin aldosterone system in clinical practice. Expert Opin Pharmacother. 2015;16(15):2283-92.

8. Xie X, Liu Y, Perkovic V, et al. Renin-angiotensin system inhibitors and kidney and cardiovascular outcomes in patients with CKD: a Bayesian network meta-analysis of randomized clinical trials. Am J Kidney Dis. 2016;67(5):728-41.

9. Zhang Y, He D, Zhang W, et al. ACE inhibitor benefit to kidney and cardiovascular outcomes for patients with non-dialysis chronic kidney disease stages 3-5: a network meta-analysis of randomised clinical trials. Drugs. 2020;80(8):797-811.

10. Epstein M, Williams $\mathrm{GH}$, Weinberger $\mathrm{M}$, et al. Selective aldosterone blockade with eplerenone reduces albuminuria in patients with type 2 diabetes. Clin J Am Soc Nephrol. 2006;1(5):940-51.

11. Minakuchi $\mathrm{H}$, Wakino $\mathrm{S}$, Urai $\mathrm{H}$, et al. The effect of aldosterone and aldosterone blockade on the progression of chronic kidney disease: a randomized placebo-controlled clinical trial. Sci Rep. 2020;10(1):16626.

12. Barrera-Chimal J, Girerd S, Jaisser F. Mineralocorticoid receptor antagonists and kidney diseases: pathophysiological basis. Kidney Int. 2019;96(2): 302-19.

13. Zhou X, Ono H, Ono Y, Frohlich ED. Aldosterone antagonism ameliorates proteinuria and nephrosclerosis independent of glomerular dynamics in L-NAME/SHR model. Am J Nephrol. 2004;24(2):242-9. 
14. Pitt B, Reichek N, Willenbrock R, et al. Effects of eplerenone, enalapril, and eplerenone/enalapril in patients with essential hypertension and left ventricular hypertrophy: the 4E-left ventricular hypertrophy study. Circulation. 2003;108(15):1831-8.

15. Zhou G, Johansson U, Peng XR, Bamberg K, Huang $Y$. An additive effect of eplerenone to ACE inhibitor on slowing the progression of diabetic nephropathy in the $\mathrm{db} / \mathrm{db}$ mice. Am J Transl Res. 2016;8(3): 1339-54.

16. Bakris GL, Agarwal R, Anker SD, et al. Effect of finerenone on chronic kidney disease outcomes in type 2 diabetes. N Engl J Med. 2020;383(23): 2219-29.

17. Ahn JH, Hong HC, Cho MJ, et al. Effect of eplerenone, a selective aldosterone blocker, on the development of diabetic nephropathy in type 2 diabetic rats. Diabetes Metab J. 2012;36(2):128-35.

18. Oudit GY, Liu GC, Zhong J, et al. Human recombinant ACE2 reduces the progression of diabetic nephropathy. Diabetes. 2010;59(2):529-38.

19. Sasaki S, Higashi Y, Nakagawa K, Matsuura H, Kajiyama G, Oshima T. Effects of angiotensin-(1-7) on forearm circulation in normotensive subjects and patients with essential hypertension. Hypertension. 2001;38(1):90-4.

20. Carver KA, Smith TL, Gallagher PE, Tallant EA. Angiotensin-(1-7) prevents angiotensin II-induced fibrosis in cremaster microvessels. Microcirculation. 2015;22(1):19-27.

21. Zimmerman D, Burns KD. Angiotensin-(1-7) in kidney disease: a review of the controversies. Clin Sci (Lond). 2012;123(6):333-46.

22. Pinheiro SV, Simoes ESAC. Angiotensin converting enzyme 2, angiotensin-(1-7), and receptor MAS axis in the kidney. Int J Hypertens. 2012;2012: 414128.

23. Paz Ocaranza M, Riquelme JA, Garcia L, et al. Counter-regulatory renin-angiotensin system in cardiovascular disease. Nat Rev Cardiol. 2020;17(2): 116-29.

24. Domenig O, Manzel A, Grobe N, et al. Neprilysin is a mediator of alternative renin-angiotensin-system activation in the murine and human kidney. Sci Rep. 2016;21(6):33678.

25. Perkins BA, Ficociello LH, Ostrander BE, et al. Microalbuminuria and the risk for early progressive renal function decline in type 1 diabetes. J Am Soc Nephrol. 2007;18(4):1353-61.

26. Chobanian AV, Bakris GL, Black HR, et al. Seventh report of the Joint National Committee on
Prevention, Detection, Evaluation, and Treatment of High Blood Pressure. Hypertension. 2003;42(6): 1206-52.

27. Pavo N, Wurm R, Goliasch G, et al. Renin-angiotensin system fingerprints of heart failure with reduced ejection fraction. J Am Coll Cardiol. 2016;68(25):2912-4.

28. Guo ZPM, McWhinney BC. Measurement of equilibrium angiotensin II in the diagnosis of primary aldosteronism. Clin Chem. 2020;66(3):483-92.

29. Guo ZPM, Cowley D, et al. Effects of ramipril on the aldosterone/renin ratio and the aldosterone/angiotensin II ratio in patients with primary aldosteronism. Hypertension. 2020;76:488-96.

30. Croghan CW, Egeghy PP. Methods of dealing with values below the limit of detection using SAS. https://analytics.ncsu.edu/sesug/2003/SD08Croghan.pdf.

31. Lubin JH, Colt JS, Camann D, et al. Epidemiologic evaluation of measurement data in the presence of detection limits. Environ Health Perspect. 2004;112(17):1691-6.

32. Kovarik JJ, Antlanger M, Domenig O, et al. Molecular regulation of the renin-angiotensin system in haemodialysis patients. Nephrol Dial Transplant. 2015;30(1):115-23.

33. Kovarik JJ, Kopecky C, Antlanger M, et al. Effects of angiotensin-converting-enzyme inhibitor therapy on the regulation of the plasma and cardiac tissue renin-angiotensin system in heart transplant patients. J Heart Lung Transplant. 2017;36(3): 355-65.

34. Cassis P, Locatelli M, Corna D, Villa S, Rottoli D, Cerullo D, et al. Addition of cyclic angiotensin(1-7) to angiotensin-converting enzyme inhibitor therapy has a positive add-on effect in experimental diabetic nephropathy. Kidney Int. 2019;96(4): 906-17.

35. Shefer G, Marcus Y, Knoll E, Dolkart O, Foichtwanger S, Nevo N, et al. Angiotensin 1-7 is a negative modulator of aldosterone secretion in vitro and in vivo. Hypertension. 2016;68(2):378-84.

36. Epstein M. Aldosterone and mineralocorticoid receptor signaling as determinants of cardiovascular and renal injury: from hans selye to the present. Am J Nephrol. 2021;52(3):209-16.

37. Weinberger $\mathrm{MH}$, Roniker B, Krause SL, Weiss RJ. Eplerenone, a selective aldosterone blocker, in mild-to-moderate hypertension. Am J Hypertens. 2002;15(8):709-16. 
38. Sato A, Fukuda S. Clinical effects of eplerenone, a selective aldosterone blocker, in Japanese patients with essential hypertension. J Hum Hypertens. 2010;24(6):387-94.

39. Shavit L, Lifschitz MD, Epstein M. Aldosterone blockade and the mineralocorticoid receptor in the management of chronic kidney disease: current concepts and emerging treatment paradigms. Kidney Int. 2012;81(10):955-68.

40. Agarwal R, Rossignol P, Garza D, Mayo MR, Warren $\mathrm{S}$, Arthur $\mathrm{S}$, et al. Patiromer to enable spironolactone use in the treatment of patients with resistant hypertension and chronic kidney disease: rationale and design of the AMBER study. Am J Nephrol. 2018;48(3):172-80.

41. Antlanger M, Bernhofer S, Kovarik JJ, Kopecky C, Kaltenecker CC, Domenig O, et al. Effects of direct renin inhibition versus angiotensin II receptor blockade on angiotensin profiles in non-diabetic chronic kidney disease. Ann Med. 2017;49(6): 525-33. 